\title{
EXPERIENCIA CUBANA EN ORGANOPÓNICOS, UNA OPCIÓN PARA LA SEGURIDAD ALIMENTARIA EN NICARAGUA
}

\author{
Mayra L. Puente Isidrón. \\ Coordinadora de Agroindustria/Departamento de Agronomía. \\ Universidad Central de Las Villas. Cuba \\ mayrapi@ucvl.edu.cu
}

\section{RESUMEN}

El desarrollo de la agricultura urbana en Cuba se fundamenta en el aprovechamiento del potencial productivo existente en cada localidad; incluyendo la fuerza laboral disponible en las ciudades y pueblos, rescatando los conocimientos heredados de los ancestros, enriquecidos con los últimos logros científicotécnicos que permiten el incremento significativo de productos agrícolas a la población. Esto contribuye a consolidar la tradición de un país netamente agrícola. Esta modalidad en la agricultura ha alcanzado gran popularidad y consiste fundamentalmente en la incorporación de la población para resolver parte de sus propios problemas alimenticios lo que implica su propia seguridad alimentaria. En este trabajo se expone los principales resultados e impactos que durante el desarrollo de la producción de alimentos se han alcanzado en Cuba, estrechamente relacionados con aspectos específicos de la nutrición humana, decisivos en la apertura económica a la crisis alimentaria de Cuba e importante aporte de vitaminas, minerales, proteína vegetal y un poco animal. Estos impactos han colaborado en la educación para la creación de nuevos hábitos alimentarios, basados en las posibilidades reales de nuestra sociedad.

\section{INTRODUCCIÓN}

El perfeccionamiento de la producción de hortalizas ha contemplado el uso de tecnologías que posibilitan el incremento del surtido y de la calidad de las mismas, permitiendo el incremento de variadas producciones y mayores ofertas en meses de altas temperaturas, alta radiación solar e intensas lluvias.

Las primeras experiencias en la aplicación del movimiento de la Agricultura Urbana en Cuba, como tecnología estratégica, se realizaron en la provincia de Villa Clara, en los municipios de Caibarién, Santa Clara y Santo Domingo, con resultados altamente favorables.

Actualmente, la carencia de fuentes de financiamiento, principalmente hacia países subdesarrollados, el predominio de la informática y otros servicios sobre la producción, la industria y la globalización como proceso resultante del desarrollo incesante de las fuerzas productivas en el mundo, constituyen un factor común, que pueden conver-

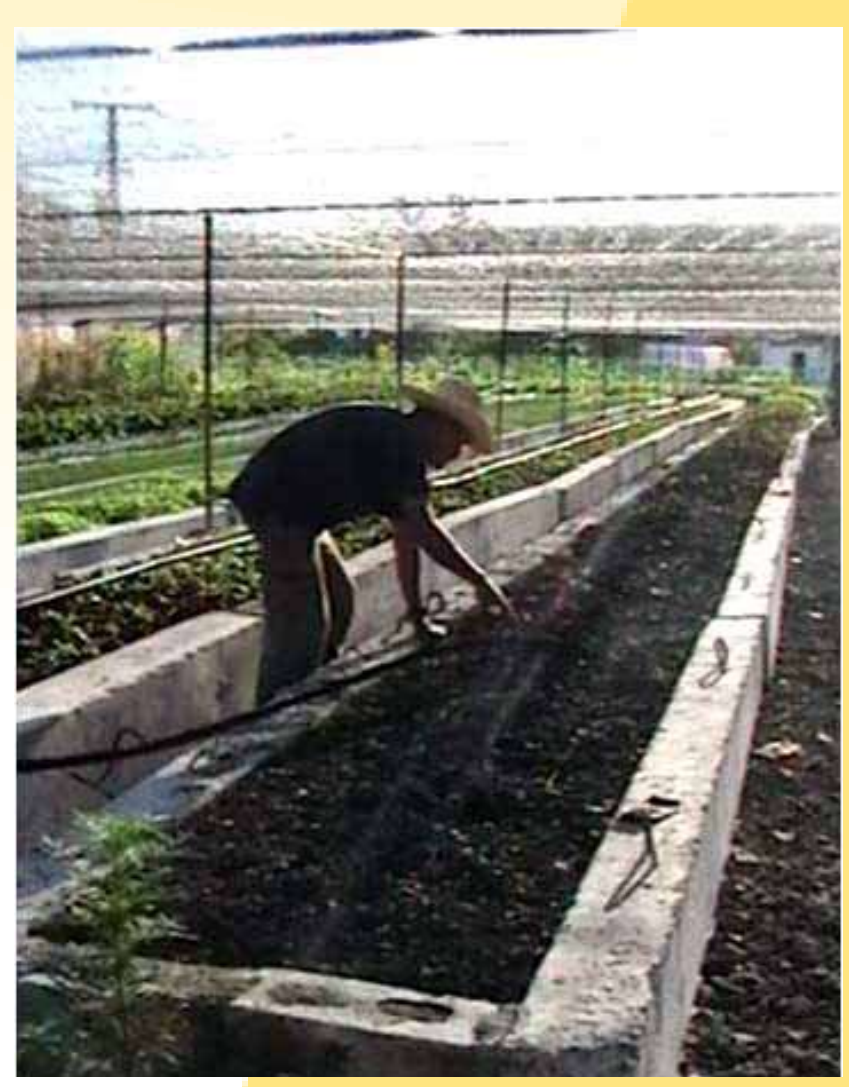

Preparación de cámara 
tirse en fuente de oportunidades o amenazas para cualquier nación u organización, de lo cual no escapa Cuba, y con el que tiene que interactuar para establecer un equilibrio dinámico. Esto demanda, para enfrentarlo, una economía de mayor apertura, competitividad y capacidad de adaptación continua a los cambios globales.

Durante muchos años la producción de hortalizas, para el consumo fresco en Cuba, se llevó a cabo bajo la dirección de las grandes empresas estatales, basada en los principios de la revolución verde, es decir, altos insumos para altos rendimientos y producir grandes volúmenes en áreas compactas de diferentes cultivos hortícolas.

La propia naturaleza de esos vegetales hacía de la transportación y el acopio, puntos vulnerables de una larga cadena de producción (acopio - transportación - acopio- puntos de venta - consumidor), llegando a los hogares apenas del 50 al $60 \%$ de los vegetales producidos y en la mayoría de los casos en condiciones de mala calidad.

\section{DESARROLLO}

La producción de hortalizas y condimentos frescos en la agricultura urbana ha mantenido un crecimiento sostenido a partir del año 1994, a un ritmo de 2-3 veces superior de un año a otro durante los primeros 4 años y de 1.8 veces durante los últimos años.

Este movimiento como programa comenzó a desarrollarse en la provincia de Villa Clara en 1994, limitado inicialmente a la producción de hortalizas

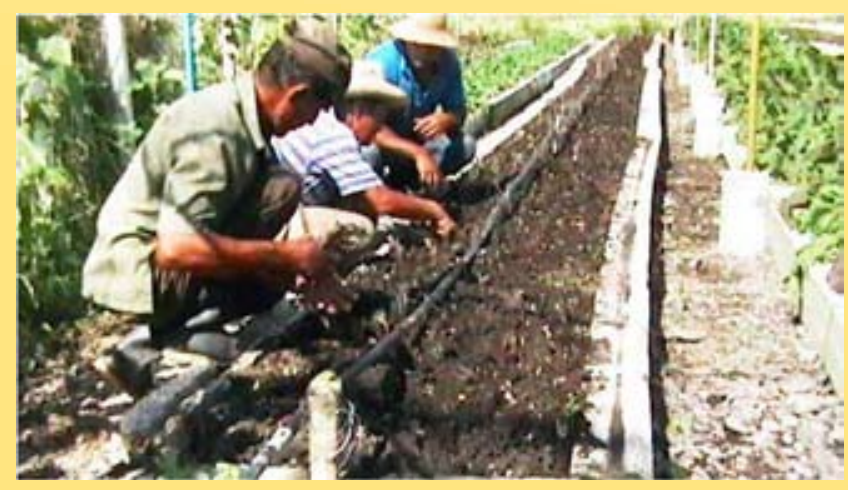

Plantación de remolacha

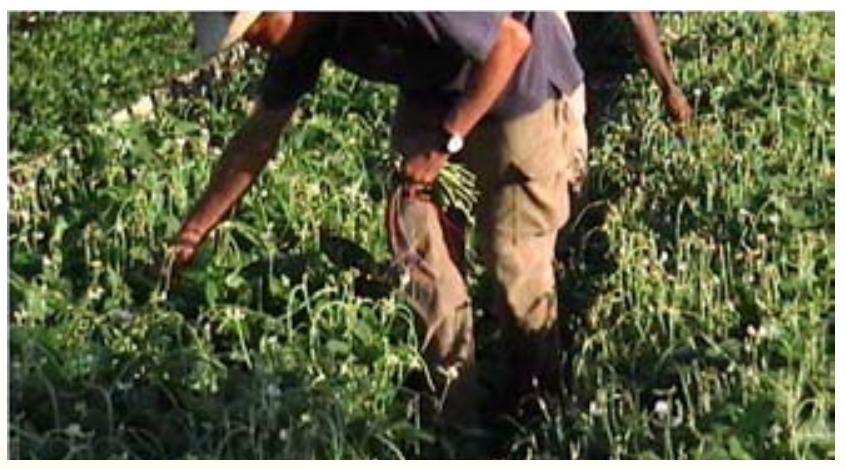

Cosecha

en huertos y organopónicos, con un mayor resultado en los municipios de Caibarién y Santa Clara, siendo su misión "Satisfacer las necesidades de nutrición de la población villaclareña, a través de la producción y comercialización de hortalizas, frutales, granos y proteínas, durante todo el año, de forma liberada a precios módicos, con la calidad exigida, de forma sana y sostenible".

Las áreas de resultados claves (ARC) constituyen el conjunto de actividades que mayores impactos provocan en el cumplimiento de tal misión. Entre las ARC definidas para este movimiento en la provincia de Villa Clara, se encuentra el "Subprograma de hortalizas, materia orgánica y semillas", cuyo objetivo estratégico es lograr no menos de 292,820 TM de hortalizas y condimentos frescos, manteniendo la diversidad en cultivos y superando la eficiencia del año anterior y que permita garantizar el consumo de 300 gramos per cápita de hortalizas diarias que recomienda la FAO.

Para cumplir con este objetivo se establecieron y desarrollaron los organopónicos y huertos intensivos como formas de producción para lograr la producción y comercialización de las hortalizas, frutales, granos y proteínas durante todo el año, garantizando la nutrición variada de la población, a precios módicos.

Estos organopónicos se caracterizan por:

- Empleo de medios biológicos sin utilización de pesticidas químicos para combatir plagas. 
- Empleo de fertilizantes orgánicos en lugar de fertilizantes químicos.

- Aprovechamiento del área en sentido horizontal y vertical, mediante un diseño previo.

- La obtención diaria de alimentos frescos, nutritivos y a muy bajos precios.

- Crear un espacio que además de útil, sea bonito y se complemente con flores y otras plantas ornamentales.

- Mejorar el ambiente por el efecto benéfico en la fauna, la temperatura y otros.

\section{Impactos de los sub-programas de la agricul- tura urbana}

La producción de hortalizas en la agricultura urbana ha mantenido un sostenido crecimiento en los últimos años, registrándose en la actualidad una producción, por metro cuadrado, de $28 \mathrm{Kg}$. Estos resultados han sido posible por el intenso trabajo en la organización de Organopónicos, Huertos Intensivos, Parcelas y Fincas Suburbanas, así como la vinculación de Patios y Huertos caseros, encontrándose en el presente 28,000 hectáreas dedicadas al cultivo de hortalizas en este programa.

Cada unidad a los distintos niveles se rige por los principios generales del Programa Nacional de Agricultura Urbana, y sus trabajadores se encuentran vinculados a la producción y al resultado final de su trabajo.

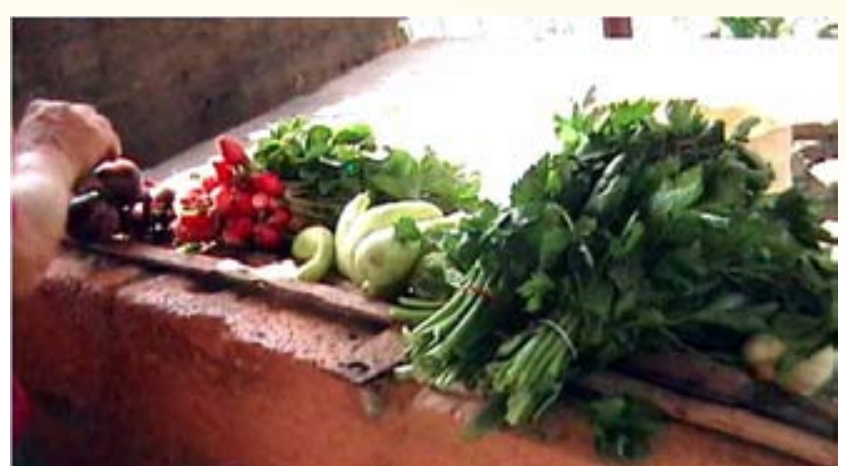

Cosecha a la venta

Las tecnologías utilizadas contemplan el uso intensivo de la materia orgánica para garantizar la nutrición de las plantas y de los controles biológicos para la protección fitosanitaria. Esto hace que los productos sean totalmente inocuos para la salud, además, resultan de una calidad biológica superior al desarrollarse de forma natural y sana.

Las principales tecnologías utilizadas para la producción de abonos orgánicos son la Lombricultura o Vermicultura y la elaboración de compost, utilizándose como materia prima distintos residuos orgánicos de origen animal, vegetal o mixto.

\section{Impactos científicos}

El desarrollo de la agricultura urbana se ha fortalecido con la existencia de un Programa de Investigaciones en las actividades de los distintos subprogramas. Las investigaciones son realizadas por todas las instituciones de los distintos ministerios relacionadas con la producción de alimentos.

El desarrollo de los proyectos ha generado múltiples resultados científico-técnicos, los cuales han tenido una rápida introducción en la práctica productiva utilizando la propia organización del Programa Nacional de la Agricultura Urbana y la participación directa de los investigadores en la asistencia técnica y la capacitación de los productores.

Entre los principales resultados se encuentran los siguientes:

- Tecnología para la construcción de Organopónicos y Huertos Intensivos.

- Tecnología de Huertos Intensivos de raíces y rizomas tropicales.

- Contribución al incremento de la biodiversidad.

- Generalización de la producción de semilla de hortalizas en condiciones tropicales, mediante la red de 176 Fincas Municipales de Semillas.

- Generalización de las tecnologías que permiten la aplicación de los principios agroecológicos para los cultivos y la cría de animales sobre bases orgánicas e intensivas.

- Generación de tecnologías para la producción de abonos orgánicos y biofertilizantes. 
- La generalización de las tecnologías de viveros tropicales y de la producción de posturas en casas de cultivo protegido.

- Se ha propiciado la recuperación de numerosas áreas ociosas que estaban convertidas en áreas contaminantes lo que ha permitido sanearlas y embellecer el medio ambiente.

- La introducción de tecnologías basadas en el uso de productos biológicos y el reciclaje de desechos ha favorecido la conservación del medioambiente y ha contribuido a restaurar el equilibrio ecológico que el abuso de agroquímicos tóxicos provocó durante decenios.

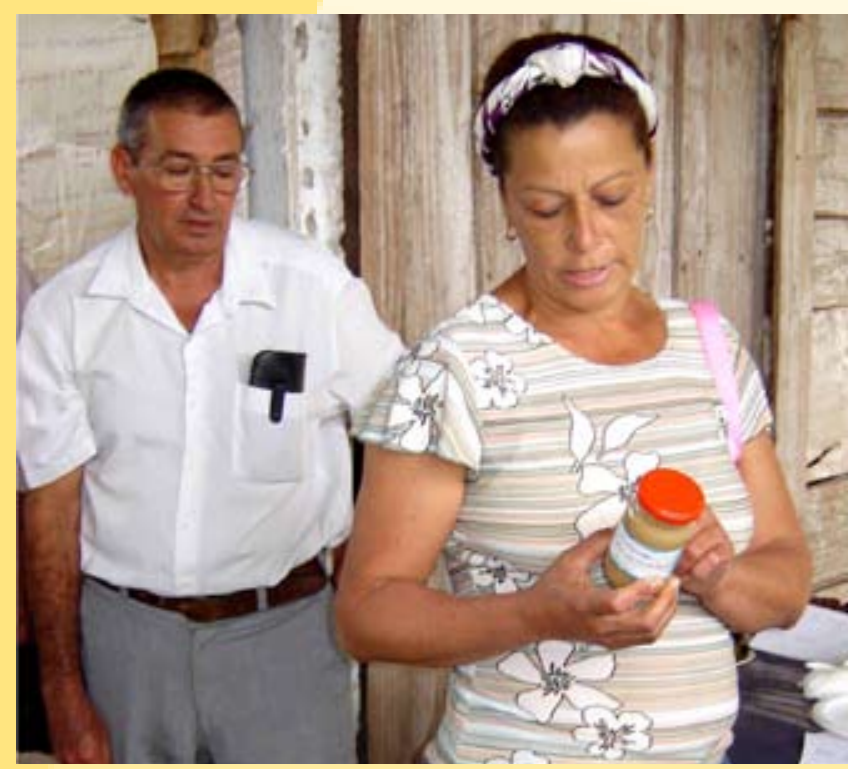

Capacitación a productores

\section{Impactos sociales y económicos}

El alcance del impacto de la agricultura urbana sobrepasa los límites de las producciones alcanzadas y de las nuevas tecnologías introducidas para perfeccionar la producción.

El rescate de tecnologías tradicionales, enriquecidas con la práctica de conceptos modernos y de las llamadas tecnologías de puntas no contaminantes ha permitido importantes avances en el incremento de la producción por unidad de área y tiempo, en el aumento de la biodiversidad y en el saneamiento de los recursos naturales, incluyendo la fertilidad del suelo.
Muchos de los logros de la agricultura urbana obtenidos en producciones en áreas pequeñas se han podido transferir a sistemas extensivos de producción, con resultados destacados.

Entre los impactos de mayor importancia social y económica se encuentran los siguientes:

- El Programa Nacional de Agricultura ha permitido la creación de más de 326 mil nuevos empleos.

Las mujeres y los jubilados encuentran en el sistema, un puesto cercano a su hogar bien remunerado y altamente estimado por la comunidad. Los jóvenes encuentran puestos de trabajo cercanos a los centros de superación técnica y cultural y con buena remuneración.

- El trabajo extensionista ha permitido a la familia combinar la producción de hortalizas, plantas medicinales para uso casero y flores para adorno con la cría de animales y los frutales.

- La generalización de las tecnologías para la pequeña agroindustria ha propiciado la capacitación de productores y amas de casa para la conservación y elaboración de alimentos. Actualmente, se procesan por esta vía 25,747 toneladas de productos diversos que se ponen a disposición de la población.

- La producción alcanzada de hortalizas y condimentos frescos ha asegurado el per cápita recomendado a sectores de mayor vulnerabilidad como son los círculos infantiles, escuelas primarias y secundarias externas, seminternas e internas, así como a los hogares de ancianos, maternos y hospitales. Hasta Mayo de 2009 se registraba un rendimiento agrícola de $28 \mathrm{Kg}$. $/ \mathrm{m}^{2}$ en esta modalidad de agricultura sostenible.

- Se ha organizado una red de círculos de interés, con participación de miles de niños en cada sub-programa de la agricultura urbana, en todos los municipios del país, con un fuerte impacto en la captación de los futuros actores en estas actividades. 
Los resultados obtenidos en este Programa Nacional lo señalan como rutas de desarrollo rural y agrícola. Cuentan con el apoyo político del Estado y de la sociedad para su consolidación y generalización en los territorios, hoy amenazados de desaparecer por los monopolios depredadores de países de América Latina y que no cuenta con el respaldo de políticas públicas favorables y de largo plazo para consolidarse como alternativa para los pequeños productores y para enfrentarse a las presiones de la agroindustria monopólica que destruye alimentos, ecosistemas, recursos genéticos y pueblos.

Con la aplicación de esta estrategia se logra la integración del proceso productivo, desde la construcción hasta la comercialización de los productos, de forma muy sencilla que lo hace apropiado para su uso en la agroindustria rural.

\section{CONCLUSIONES}

La aplicación y desarrollo del movimiento popular de Agricultura Urbana como estrategia, ha posibilitado la obtención de considerables volúmenes de producción de hortalizas, capaces de proporcionar salud y bienestar a la población y de generar altos ingresos a la economía nacional con la utilización de bajas cantidades de insumos en condiciones de agroindustria lo que constituye una estrategia favorable .

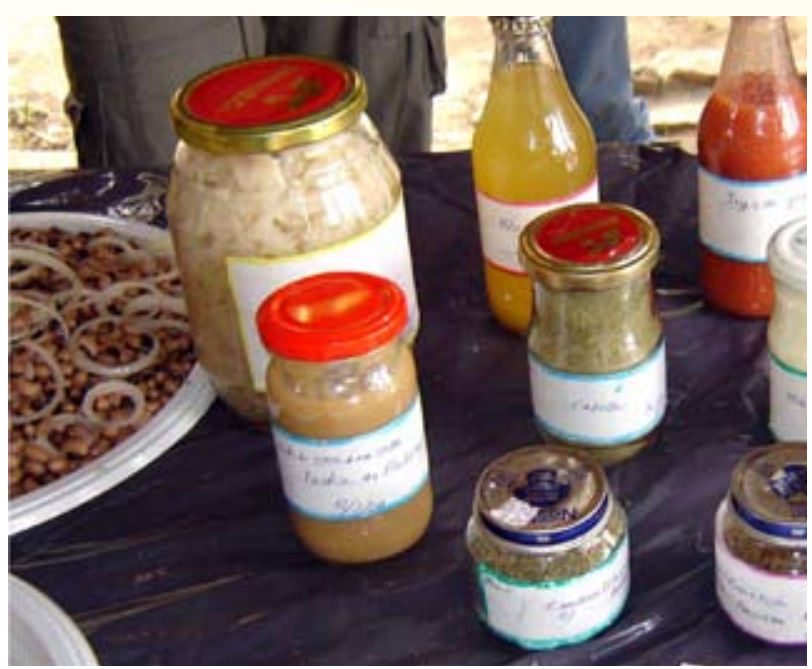

Los impactos generados por la aplicación de esta estrategia, ha permitido importante avances en el incremento de la producción por unidad de área y tiempo, en el aumento de la biodiversidad, el saneamiento de los recursos naturales así como el bienestar social de la población.

La experiencia cubana en Organopónicos para la seguridad alimentaria en la población, puede ser considerada una opción en Nicaragua, que apoye el cumplimiento de los objetivos declarados en los Proyectos SAN de Plan Nicaragua y Nutrinet de "Seguridad Alimentaria y Agricultura Sostenible".

\section{BIBLIOGRAFÍA}

1. Companioni, N. Rodríguez, A. Mirian Carrión. Yanet Ojeda. Elizabet Peña. (1999). Organopónicos y la Producción de Alimentos en la Agricultura Urbana. Seminario Taller República de Cuba MINAGRIC.

2. Companioni, N. Rodríguez, A. Mirian Carrión. Yanet Ojeda. Elizabet Peña. (2001). La Agricultura Urbana en Cuba. Transformando el campo cubano, avances de la agricultura sostenible, Habana.

3. Carrión, Mirian. (1996). Agricultura del Hogar en la Agricultura Urbana, Desarrollo Sostenible. MINAGRIC, FIDA, CIARA. Pág. $58-72$.

4. Cuba: La agricultura orgánica puede contribuir a resolver la crisis alimentaria. http://acsur.org.

5. FAO. (2000). Mejorando la nutrición a través de huertos y granjas familiares. Roma. Pág. 1 a la 239.

6. González, F. (2001). Curso de Dirección Estratégica. Facultad de Ingeniería Industrial y Economía, Villa Clara. Grupo Nacional de la Agricultura Urbana. (2002). Lineamientos para los subprogramas de la Agricultura Urbana. pp 96.

7. G. Kastler, 2008. Planet Diversity. Bonn

8. López, F. (2002). Agricultura Urbana Locomotora Eficiente en la Producción de Alimentos. Pág. 4 y 5.

9. Proyectos de SAN de Plan Nicaragua han beneficiado a 300 familias de 24 comunidades. 2009 . Disponible en: http://NicNet/NutriNetNICARAGUA. 